\title{
The dykes in the western fringe of the Ybytyruzú Hills, central-eastern Paraguay region
}

\section{Os diques na encosta ocidental da Serra de Ybytyruzú, região centro-oriental do Paraguai}

\author{
Celso de Barros Gomes ${ }^{1}$, Ana Maria Castillo Clerici², Victor Velázquez Fernandez ${ }^{3}$, \\ Angelo De $\mathrm{Min}^{4}$, Piero Comin-Chiaramonti ${ }^{4}$ \\ ${ }^{1}$ Institute of Geosciences, Universidade de São Paulo - USP, Rua do Lago, 562, \\ Butantã, CEP 05508-080, São Paulo, SP, BR (cgomes@usp.br) \\ ${ }^{2}$ Technical Coordinator attached. Framework Program La Plata Basin, Buenos Aires, Argentina (ana.clerici@gmail.com) \\ ${ }^{3}$ School of Arts, Sciences and Humanities, Universidade de São Paulo - USP, São Paulo, SP, BR (vvf@usp.br) \\ ${ }^{4}$ Department of Mathematics and Geosciences, University of Trieste, Trieste, Italy (demin@univ.trieste.it; comin@units.it)
}

Received on October $15^{\text {th }}, 2016$; accepted on April $5^{\text {th }}, 2017$

\begin{abstract}
The Cordillera del Ybytyruzú region of central-eastern Paraguay is predominantly formed by tholeiitic lava flows from the Early Cretaceous age (130-134 Ma) cut by K-alkaline dykes and intrusive bodies aged between 125 and $127 \mathrm{Ma}$. Petrochemical studies focusing on 14 samples of dykes from the region show an apparent affinity to Roman Province Type rocks and total consistency with the overall petrographic association of the Asunción-Sapucai-Villarrica (ASV) graben. Two main potassic suites are distinguished: B-P (basanite-tephrite-phonotephrite-phonolite) and AB-T (alkali basalt-trachybasalt-trachyandesite-trachyphonolite/trachyte).The occurrence of lamproitic rocks in the area has so far not been confirmed.
\end{abstract}

Keywords: Ybytyruzú; Eastern Paraguay; Potassic Alkaline Dykes.

\section{Resumo}

A Cordilheira do Ybytytyruzú na região centro-oriental do Paraguai é formada dominantemente de derrames de lavas toleíticas de idade Cretáceo Inferior (130-134 Ma) cortados por diques alcalinos potássicos e corpos intrusivos com idades entre 125 e $127 \mathrm{Ma}$. Estudos petroquímicos focalizando 14 amostras de diques da região mostram uma aparente afinidade com as rochas da Província do Tipo Romano e total consistência com a associação petrográfica global do gráben Assunção-Sapucai-Villarrica (ASV). Duas suítes potássicas principais são distinguidas: B-P (basanito-tefrito-fonotefrito-fonolito) e AB-T (álcali basalto-traquibasalto-traquiandesito-traquifonolito/traquito). A ocorrência de rochas lamproíticas na área não foi até então confirmada.

Palavras-chave: Ybytyruzú; Paraguai Oriental; Diques Alcalino-potássicos. 


\section{INTRODUCTION}

Eastern Paraguay represents the westernmost fringe of Early Cretaceous Paraná flood tholeiites: the Serra Geral Formation (SGF), also known as the Alto Paraná Formation, in Paraguayan territory, aged 130-134 Ma, according to Piccirillo and Melfi (1988). It has been the site of multiple episodes of alkaline magmatism of sodic and potassic composition occurring during the Triassic, Early Cretaceous (pre- and post-dating the SGF volcanic rocks) and Paleocene times (Comin-Chiaramonti and Gomes, 1996, 2005; Comin-Chiaramonti et al., 2007a, 2007b; Gomes et al., 2013).

Geophysical studies show that Eastern Paraguay is cut by two sets of major crustal-scale faults, an older NE-trending set inherited from the Precambrian, and a younger NW-trending set. The latter defines at least four NW-trending graben system or fault-controlled basins that formed in late Mesozoic as response to NE-SW direct extension and continued evolving into upper Tertiary times. One of these graben systems has been identified in Southeastern Paraguay by gravity data and Landsat image analysis, the Asunción-Sapucai-Villarrica (ASV), and represents the most apparent rift structure in the country (Comin-Chiaramonti et al., 1999). Geology and gravity data indicate that this tectonic feature extends at least $200 \mathrm{~km}$ from Asunción southeasterly to Paraguari, varying from 25 to $40 \mathrm{~km}$ wide, and from there along an EW to ESE trajectory to at least the Ybytyruzú hills, east of the city of Villarrica (Figures 1A and 1B). In the ASV area, the Mesozoic Cordillera del Ybytyruzú tholeiitic flows occur alongside potassic alkaline complexes and dykes of the same age as well as more recent Na-alkaline plugs and subordinate dykes.

Based on 527 chemical analyses of silicate rocks (intrusives, effusives and dykes) from the ASV graben, Comin-Chiaramonti et al. (1996a, 1996b, 1996c, 1996e) proposed their inclusion into two major groups: potassic and sodic. Of these, 220 analyses correspond to a dyke swarm previously investigated thoroughly by Gomes et al. (1989), Comin-Chiaramonti et al. (1992, 2013) and Velázquez et al. (2011). The more recent alkaline sodic rocks outcrops in the area (e.g., Cerro Medina, Cerro Gimenez) are notably peralkaline in composition. In the $R_{1}-R_{2}$ diagram (De La Roche et al., 1980), two main suites of potassic rocks can be distinguished on Figure 2, conforming to the general trends of basanite to phonolite (B-P) and alkaline basalt to trachyphonolite/trachyte (AB-T) suites for the whole graben. Petrochemical evidence, in addition to textural and mineralogical features, point to the fractional crystallization process as potentially important in the evolution of ASV rocks (Comin-Chiaramonti et al., 1996a, 1996b, 1996c, 1996d, 1997). Based on the peralkaline index $\left(\mathrm{K}_{2} \mathrm{O}+\mathrm{Na}_{2} \mathrm{O} /\right.$ $\mathrm{Al}_{2} \mathrm{O}_{3}$ molar) the potassic ASV rock types are clearly plotted into the Roman Province Type (RPT) lava field of Barton (1979) and Foley (1992).
On the other hand, Presser et al. (2014), based on mineralogical associations and mineral compositions, have classified some Ybytyruzú and Mbocayaty (an alkaline complex occurring close to the Cordillera, see Figure 1B) volcanic rocks bearing phenocrysts of olivine, phlogopite, diopside, opaques and occasional leucite as phlogopite and leucite lamproites. However, because Comin-Chiaramonti and Gomes (1996) did not recognize any rock types of lamproitic affinity [(i.e., rocks fulfilling the following conditions: molar ratios $\mathrm{K}_{2} \mathrm{O} /$ $\mathrm{Na}_{2} \mathrm{O}>3, \mathrm{~K}_{2} \mathrm{O} / \mathrm{Al}_{2} \mathrm{O}_{3}>1$, and $\left(\mathrm{K}_{2} \mathrm{O}+\mathrm{Na}_{2} \mathrm{O}\right) / \mathrm{Al}_{2} \mathrm{O}_{3}>1$; contents of $\mathrm{Ba}>2000, \mathrm{Zr}>500$ and $\mathrm{La}>200 \mathrm{ppm}$; all data according to Le Maitre (1976), Bergman (1987), Woolley et al. (1996), and Mitchell and Bergman (1991)], in the entire ASV graben. Examining the Cordillera del Ybytyruzú and Mbocayaty dyke occurrences in more detail was judged convenient.

\section{CORDILLERA DEL YBYTYRUZÚ}

The Cordillera del Ybytyruzú comprises a highland of Paraná tholeiitic flows (Bellieni et al., 1986), with local K-alkaline dyke intrusions (sps. 1 to 7 and 10-11 of Figure 3). The volcanic rocks form a thick sequence up to $800 \mathrm{~m}$ high of several tens of lava flows with small red sandstone intercalations (Triassic-Jurassic Misiones Formation sediments, Comin-Chiaramonti and Gomes, 1996). According to high quality ${ }^{40} \mathrm{Ar} /{ }^{39} \mathrm{Ar}$ determinations (Renne et al., 1993) and paleomagnetic data (Ernesto et al., 1996; Comin-Chiaramonti et al., 2007a, 2007b), the best origin period for the basaltic rocks overall is between 130 and 134 Ma. Some K-alkaline complexes, e.g., Cerro E Santa Helena, Cerro km 23 and Cerro San Benito, crop out at the southwestern margins of the Cordillera (Figure 3).

\section{Cerro E Santa Helena}

Cerro E Santa Helena is an oval shaped complex $320 \mathrm{~m}$ high, covers an area of $3.3 \mathrm{~km}^{2}$ and contains intrusive (gabbros, essexitic gabbros and essexites) and effusive (K-tephrites, basanites and subordinate alkali basalts) rock types. The ${ }^{40} \mathrm{Ar} /{ }^{39} \mathrm{Ar}$ plateau age of biotite from the essexitic rock is $125.6 \pm 0.2 \mathrm{Ma}$ (Comin-Chiaramonti et al., 2007a, 2007b). Two dykes, a Na-alkali basalt (sp. 8) and a K-alkaline trachyphonolite (sp. 9) are indicated in Figure 3.

\section{Cerro Km 23}

This body forms a massive, circular stock $275 \mathrm{~m}$ high with an area $0.8 \mathrm{~km}^{2}$, and occurs approximately $1 \mathrm{~km} \mathrm{NE}$ of the Roque G. de Santa Cruz village (Figure 3). The main rock-types are K-essexitic gabbros and essexites aged $127.7 \pm 0.1 \mathrm{Ma}$ $\left({ }^{40} \mathrm{Ar} /{ }^{39} \mathrm{Ar}\right.$ plateau age of biotite from an essexitic gabbro, $\mathrm{cf}$. Comin-Chiaramonti et al., 2007a, 2007b). A phonotephritic dyke is also present (sp. 12 of Figure 3). 


\section{Cerro San Benito}

The outcrop is represented by a massive oval-shaped 230-m high stock, covering an area of $0.3 \mathrm{~km}^{2}$ and cut by a trachybasalt dyke (sp. 14 of Figure 3). The rock types are K-essexites and essexitic gabbros. $\mathrm{A}^{40} \mathrm{Ar} /{ }^{39} \mathrm{Ar}$ plateau age of $127.4 \pm 0.3 \mathrm{Ma}$ in biotite is given by Comin-Chiaramonti et al. (2007a, 2007b).

\section{MBOCAYATY}

An elliptic stock covering an area of $1.5 \mathrm{~km}^{2}$ crops out at the Mbocayaty village (sp. 6 of Figure 1B), located on the west side of the Ybytyruzú Hills and approximately $5 \mathrm{~km} \mathrm{NE}$ of the town of Villarrica. The main rock-types are essexitic gabbros and essexites of potassic affinity. Velázquez et al.
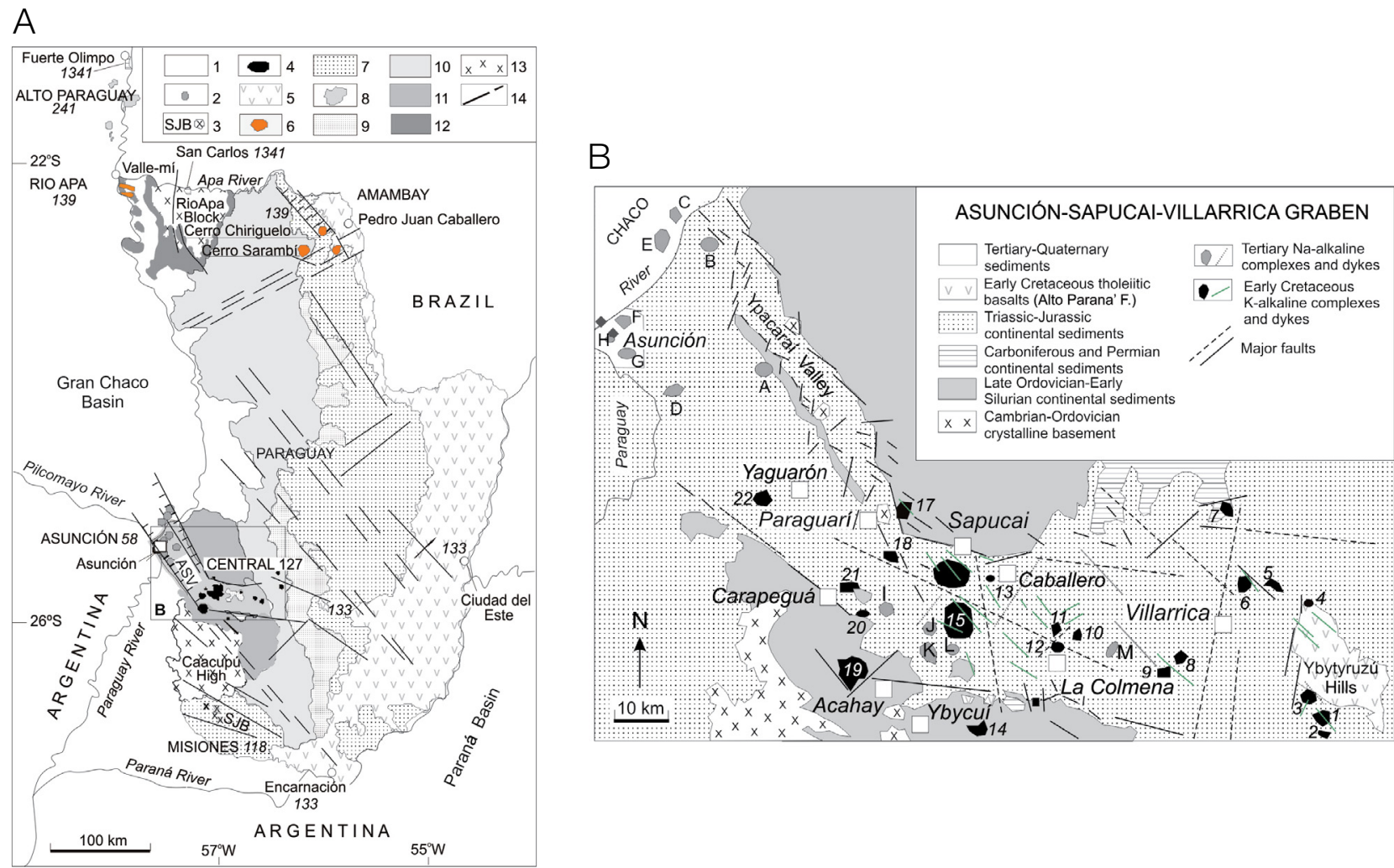

Figure 1. (A) Geological sketch-map of Eastern Paraguay (modified and simplified after Comin-Chiaramonti and Gomes, 1996, 2005). ASV: Asunción-Sapucai-Villarrica graben. Legends: 1, Neogene and Paleogene sedimentary cover (i.e., Gran Chaco sediments); 2, Paleogene sodic alkaline rocks, Asunción Province; 3, Late Early Cretaceous sodic alkaline rocks (Misiones Province; San Juan Bautista, SJB); 4, Early Cretaceous potassic alkaline rocks (post-tholeiites, Central Province); 5, Early Cretaceous Paraná Basin tholeiites; 6, Early Cretaceous potassic alkaline rocks (pre-tholeiites, Apa and Amambay Provinces); 7, Triassic-Jurassic sedimentary rocks (Misiones Formation); 8, Permo-Triassic alkaline rocks (Alto Paraguay Province); 9, Permian sedimentary rocks (Independencia Group); 10, Permo-Carboniferous sedimentary rocks (Coronel Oviedo Group); 11, Ordovician-Silurian sedimentary rocks (Caacupé and Itacurubí Groups); 12, Carbonates from a Cambro-Ordovician platform (Itacupumí Group); 13, Archean to early Paleozoic crystalline basement; 14, Major tectonic lineaments and faults. Quoted ages (Ma) refer to ${ }^{40} \mathrm{Ar} /{ }^{39} \mathrm{Ar}$ plateau ages for the main magmatic rock-types (rhyolitic: Gomes et al., 2000; tholeiitic: Renne et al., 1992, 1993; alkaline: Comin-Chiaramonti et al., 2007a, 2007b). (B) Geological sketch map of the Asunción-Sapucai-Villarrica graben showing the main occurrences of alkaline rocks (modified after Comin-Chiaramonti et al., 2013). In parentheses preferred K/Ar and plateau ${ }^{40} \mathrm{Ar} /{ }^{39} \mathrm{Ar}$ ages (in bold) in Ma according to Comin-Chiaramonti et al. (2007a, 2007b) for some complexes. K-alkaline complexes: 1, Cerro Km 23 (132-128 Ma); 2, Cerro San Benito (127 Ma); 3, Cerro E Santa Elena (126 Ma); 4, Northwestern Ybytyruzú (125-129 Ma); 5, Cerro Capiitindy (not available); 6, Mbocayaty (126-130, 126); 7, Aguapety Portón (128-133, 126); 8, Cerro Itapé (126); 9, Cerrito Itapé (not available); 10, Cerro Cañada (127); 11, Cerro Chobí (127); 12, Potrero Garay (not available); 13, Catalán (127.); 14, Cerro San José (127); 15, PotreroYbaté (126-128, 128); 16, Sapucai (119-131, 126); 17, Cerro Santo Tomás (126-130, 127); 18, Cerro Porteño (not available); 19, Cerro Acahay (118, 127); 20, Cerro Pinto (127); 21, Cerro Ybypyté (124); 22, Cerro Arrúa-í (126-132, 129). Na-alkaline complexes: A, Cerro Patiño (39); B, Limpio (50); C, Cerro Verde (57, 61); D, Cerro Ñemby (46, 61); E, Cerro Confuso (55-61); F, NuevaTeblada (46-57); G, Lambaré (49); H, Cerro Tacumbú (41-46, 58); I, Cerro Yariguaá (58); J, Cerrito (56); K, Cerro Gimenez (66); L, Cerro Medina (67); M, Colonia Vega (68). Detailed information on individual occurrences, such as geological map, location, country rocks, forms, and main rock types is provided in Comin-Chiaramonti et al., 1996d. 
(1992) reported a $\mathrm{Rb} / \mathrm{Sr}$ isochron (biotite, feldspar and whole rock) age of $127.8 \pm 0.2 \mathrm{Ma}$ for the occurrence. Trachybasalt dykes are also present.

\section{PETROCHEMICAL AND PETROGRAPHICAL NOTES}

Major (oxide \%) and trace elements (ppm) of the dykes were carried out at the University of Trieste, Italy, by X-ray fluorescence techniques using an automatic Philips PW1400 spectrometer and pressed-powder pellets. $\mathrm{FeO}$ was determined chemically employing ammonium metavanadare. The preparation methods and analytical procedures have been described in detail by Bellieni et al. (1983).

Chemical analyses and nomenclature of the Mbocayaty and Ybytyruzú dykes are listed in Table 1. The compositions are plotted in the $\mathrm{R}_{1}-\mathrm{R}_{2}$ classification diagram (Figure 2), from which it is apparent that both suites recognized in the graben area are present. As noted in Figure 4, the rocks are predominantly potassic; three samples are transitional in composition (sps. 7,13 and 14), and only one (sp. 8) is sodic. Notably, an ocellus found in trachyandesite (sp. 2, Figure 5) appears to be highly potassic; the analyzed sample (2A) is also distinguished by its ultrabasic composition and high $\mathrm{Cr}$ and $\mathrm{Ni}$ content (Table 1). General petrographic data on these rocks are reported in Comin-Chiaramonti and Gomes (1996) and Comin-Chiaramonti et al. (1996c).

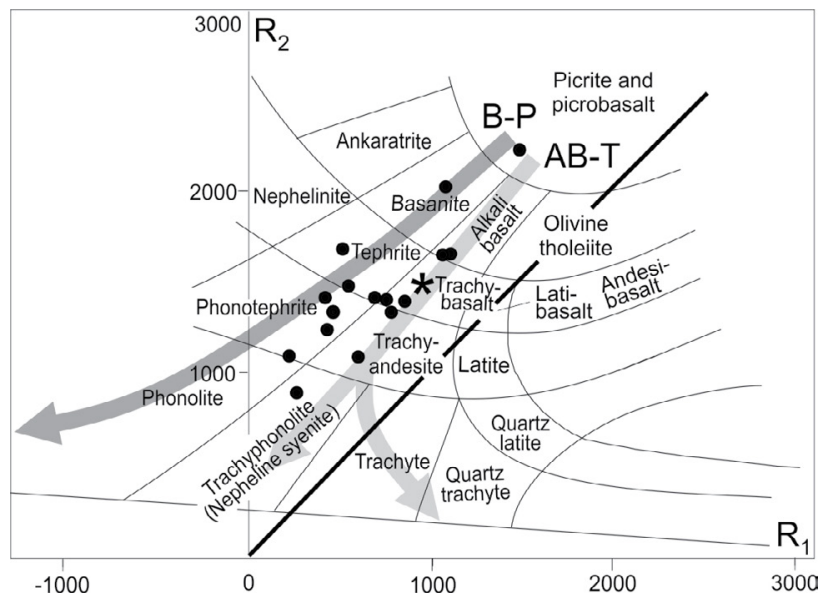

Figure 2. Plot of the analyzed dykes in the $R_{1}-R_{2}$ diagram (De La Roche et al., 1980). Symbols: full circles, Ybytyruzú samples; asterisk, Mbocayaty sample. Legends: $\mathrm{R}_{1}=4 \mathrm{Si}-1(\mathrm{Na}+\mathrm{K})-2(\mathrm{Fe}+\mathrm{Ti})$ and $\mathrm{R}_{2}=6 \mathrm{Ca}+2 \mathrm{Mg}+\mathrm{Al}$. General trends for potassic rocks of both suites (B-P, basanite-tephrite-phonotephrite-phonolite; $A B-T$, alkali basalt-trachybasalt-trachyandesite-trachyphonolite/ trachyte) of ASV graben are shown based on Comin-Chiaramonti et al. (1996c, 1997).

\section{Sodic group}

This group is represented in the Cordillera del Ybytyruzú by only one dyke of alkali basaltic composition occurring at the northern side of the Cerro E Santa Helena Complex (sp. 8 of Figure 3). The rock is texturally porphyritic with hyaline groundmass. Pheno- and microphenocrysts consist of clinopyroxene, biotite, sanidine, opaques and apatite; carbonate patches and olivine ghosts are also present.

\section{Potassic group}

\section{$B-P$ suite (basanite to phonolite)}

According to Figure 2 and Table 1, the dykes of the suite comprise one basanite (sp. 11), three tephrites (sps. 7, 12 and 13)

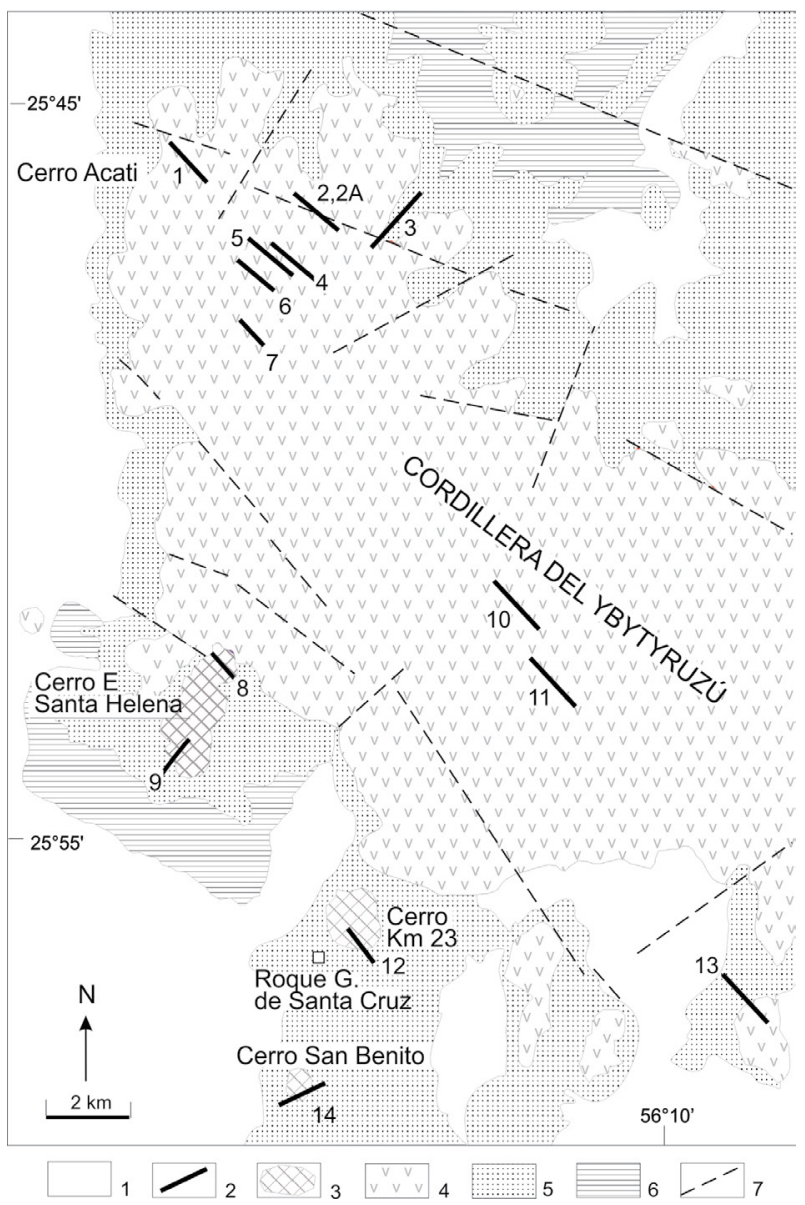

Figure 3. Geological sketch map showing the western fringe of the Cordillera del Ybytyruzú (cf. Figure 1, and page 278 of Comin-Chiaramonti and Gomes, 1996) and the location of the analyzed samples. Legends: 1: Quaternary sediments; 2: Dykes; 3: Alkaline bodies; 4: Tholeiitic flows of the Alto Paraná Formation (Early Cretaceous); 5: Sediments of the Misiones Formation (Triassic-Jurassic); 6: Sediments of the Independencia Group (Permian); 7: Major faults. 
Table 1. Chemical analyses, major and trace elements (Mbocayaty; Ybytyruzú highlands, 1-14; 2a, ocellus) of the investigated dykes. Nomenclature according to the $\mathrm{R}_{1}-\mathrm{R}_{2}$ diagram (after De La Roche et al., 1980). Other parameters: $\mathrm{mg}=\mathrm{MgO} /(\mathrm{MgO}+\mathrm{FeO})$, molar ratio; A.I., agpaitic index $\left(\mathrm{Na}_{2} \mathrm{O}+\mathrm{K}_{2} \mathrm{O}\right) / \mathrm{Al}_{2} \mathrm{O}_{3}$, molar ratio (cf. MacDonald, 1974); geographic position, direction and thickness of the bodies.

\begin{tabular}{|c|c|c|c|c|c|c|c|c|}
\hline \multirow[b]{2}{*}{ Sample } & Mbocayaty & 1 & 2 & $2 \mathrm{~A}$ & 3 & 4 & 5 & 6 \\
\hline & Trachybasalt & $\begin{array}{l}\text { Phono- } \\
\text { tephrite }\end{array}$ & Trachyandesite & Picrobasalt & $\begin{array}{l}\text { Phono- } \\
\text { tephrite }\end{array}$ & Trachyandesite & $\begin{array}{l}\text { Phono- } \\
\text { tephrite }\end{array}$ & Trachybasalt \\
\hline \multicolumn{9}{|l|}{$\mathrm{Wt} \%$} \\
\hline $\mathrm{SiO}_{2}$ & 50.31 & 52.99 & 52.90 & 41.26 & 53.11 & 51.24 & 52.46 & 50.85 \\
\hline $\mathrm{TiO}_{2}$ & 1.61 & 1.59 & 1.50 & 2.15 & 1.21 & 1.52 & 1.53 & 1.52 \\
\hline $\mathrm{Al}_{2} \mathrm{O}_{3}$ & 12.96 & 14.35 & 13.72 & 8.90 & 15.89 & 13.87 & 14.02 & 13.46 \\
\hline $\mathrm{Fe}_{2} \mathrm{O}_{3}$ & 2.11 & 5.16 & 3.90 & 2.20 & 2.40 & 3.66 & 2.66 & 3.64 \\
\hline $\mathrm{FeO}$ & 5.86 & 3.33 & 4.30 & 11.26 & 4.50 & 4.83 & 5.18 & 4.88 \\
\hline $\mathrm{MnO}$ & 0.13 & 0.19 & 0.16 & 0.26 & 0.14 & 0.15 & 0.14 & 0.15 \\
\hline $\mathrm{MgO}$ & 7.44 & 4.48 & 3.19 & 17.00 & 2.61 & 5.26 & 5.29 & 5.95 \\
\hline $\mathrm{CaO}$ & 8.13 & 6.60 & 6.03 & 11.47 & 6.00 & 7.57 & 6.51 & 7.94 \\
\hline $\mathrm{Na}_{2} \mathrm{O}$ & 3.03 & 3.88 & 3.27 & 0.47 & 4.21 & 3.34 & 3.65 & 3.01 \\
\hline $\mathrm{K}_{2} \mathrm{O}$ & 4.44 & 6.28 & 6.45 & 2.95 & 6.87 & 5.10 & 6.50 & 5.19 \\
\hline $\mathrm{P}_{2} \mathrm{O}_{5}$ & 0.47 & 0.55 & 0.53 & 0.13 & 0.57 & 0.52 & 0.54 & 0.54 \\
\hline LOI & 2.85 & 0.60 & 3.91 & 1.60 & 2.27 & 2.75 & 1.33 & 2.63 \\
\hline Sum & 99.34 & 100.00 & 99.86 & 99.65 & 99.78 & 99.61 & 99.81 & 99.76 \\
\hline \multicolumn{9}{|l|}{ ppm } \\
\hline $\mathrm{Cr}$ & 445 & 20 & 34 & 2275 & 36 & 223 & 170 & 270 \\
\hline $\mathrm{Ni}$ & 119 & 17 & 17 & 687 & 20 & 52 & 44 & 65 \\
\hline $\mathrm{Rb}$ & 80 & 112 & 119 & 58 & 138 & 88 & 127 & 89 \\
\hline $\mathrm{Sr}$ & 1550 & 946 & 857 & 420 & 1736 & 1385 & 1253 & 1432 \\
\hline $\mathrm{Zr}$ & 298 & 478 & 370 & 113 & 287 & 211 & 207 & 213 \\
\hline Y & 17 & 18 & 28 & 19 & 24 & 22 & 21 & 23 \\
\hline $\mathrm{Nb}$ & 39 & 66 & 57 & 15 & 52 & 37 & 38 & 38 \\
\hline $\mathrm{Ba}$ & 1606 & 1471 & 1391 & 1555 & 1816 & 1629 & 1610 & 1531 \\
\hline Th & 132 & 11.8 & 105 & 13.8 & 14.5 & 122 & 12.9 & 126 \\
\hline La & 82 & 162 & 147 & 45 & 128 & 100 & 99 & 96 \\
\hline $\mathrm{Ce}$ & 147 & 299 & 261 & 81 & 222 & 185 & 173 & 174 \\
\hline $\mathrm{Nd}$ & 50 & 108 & 105 & 35 & 87 & 75 & 71 & 73 \\
\hline $\mathrm{Sm}$ & 8.5 & 22 & 16 & 5.4 & 17.5 & 11.4 & 14.2 & 12.4 \\
\hline $\mathrm{Yb}$ & 1.3 & 3.2 & 2.3 & 0.8 & 2.6 & 1.7 & 2.1 & 1.8 \\
\hline $\begin{array}{l}\text { Lat } \\
\text { Long }\end{array}$ & $\begin{array}{l}25^{\circ} 42.5^{\prime} \\
56^{\circ} 25.0^{\prime}\end{array}$ & $\begin{array}{l}25^{\circ} 45.9^{\prime} \\
56^{\circ} 17.1^{\prime}\end{array}$ & $\begin{array}{l}25^{\circ} 47.9^{\prime} \\
56^{\circ} 17.1^{\prime}\end{array}$ & $\begin{array}{l}25^{\circ} 47.9^{\prime} \\
56^{\circ} 17.1^{\prime}\end{array}$ & $\begin{array}{l}25^{\circ} 46.8 \\
56^{\circ} 16.5^{\prime}\end{array}$ & $\begin{array}{l}25^{\circ} 47.2^{\prime} \\
56^{\circ} 15.7^{\prime}\end{array}$ & $\begin{array}{l}25^{\circ} 47.3^{\prime} \\
56^{\circ} 15.8^{\prime}\end{array}$ & $\begin{array}{l}25^{\circ} 47.4^{\prime} \\
56^{\circ} 15.9^{\prime}\end{array}$ \\
\hline $\begin{array}{l}\text { Thickness } \\
\text { Direction }\end{array}$ & $\begin{array}{l}2.5 \mathrm{~m} \\
\text { N30W }\end{array}$ & $\begin{array}{l}2.5 \mathrm{~m} \\
\mathrm{~N} 27 \mathrm{~W}\end{array}$ & $\begin{array}{l}2.5 \mathrm{~m} \\
\mathrm{~N} 27 \mathrm{~W}\end{array}$ & $\begin{array}{c}0.03 \mathrm{~m} \\
-\end{array}$ & $\begin{array}{l}3.0 \mathrm{~m} \\
\mathrm{~N} 30 \mathrm{~W}\end{array}$ & $\begin{array}{l}4.1 \mathrm{~m} \\
\mathrm{~N} 45 \mathrm{~W}\end{array}$ & $\begin{array}{l}3.5 \mathrm{~m} \\
\mathrm{~N} 45 \mathrm{~W}\end{array}$ & $\begin{array}{l}2.0 \mathrm{~m} \\
\mathrm{~N} 45 \mathrm{~W}\end{array}$ \\
\hline mg\# & 0.693 & 0.538 & 0.458 & 0.727 & 0.448 & 0.573 & 0.591 & 0.602 \\
\hline A.I. & 0.755 & 0.918 & 0.901 & 0.446 & 0.904 & 0.794 & 0.930 & 0.785 \\
\hline $\mathrm{R}_{1}$ & 980 & 421 & 599 & 1468 & 221 & 770 & 429 & 839 \\
\hline $\mathrm{R}_{2}$ & 1493 & 1210 & 1072 & 2245 & 1083 & 1343 & 1234 & 1409 \\
\hline
\end{tabular}


Table 1. Continuation.

\begin{tabular}{|c|c|c|c|c|c|c|c|c|}
\hline \multirow[b]{2}{*}{ Sample } & 7 & 8 & 9 & 10 & 11 & 12 & 13 & 14 \\
\hline & Tephrite & $\begin{array}{l}\text { Alkali } \\
\text { basalt }\end{array}$ & $\begin{array}{l}\text { Nepheline } \\
\text { syenite }\end{array}$ & Trachybasalt & Basanite & Tephrite & Tephrite & $\begin{array}{l}\text { Alkali } \\
\text { basalt }\end{array}$ \\
\hline \multicolumn{9}{|l|}{ Wt\% } \\
\hline $\mathrm{SiO}_{2}$ & 50.83 & 46.35 & 57.05 & 51.16 & 45.04 & 48.26 & 48.01 & 49.93 \\
\hline $\mathrm{TiO}_{2}$ & 2.00 & 1.41 & 0.65 & 1.60 & 1.76 & 1.47 & 1.61 & 1.45 \\
\hline $\mathrm{Al}_{2} \mathrm{O}_{3}$ & 10.95 & 12.65 & 17.23 & 13.63 & 11.48 & 14.70 & 14.50 & 11.63 \\
\hline $\mathrm{Fe}_{2} \mathrm{O}_{3}$ & 4.68 & 5.82 & 1.37 & 2.52 & 5.00 & 3.67 & 4.00 & 2.94 \\
\hline $\mathrm{FeO}$ & 3.90 & 4.62 & 3.00 & 6.38 & 6.34 & 5.80 & 5.21 & 6.12 \\
\hline $\mathrm{MnO}$ & 0.13 & 0.23 & 0.12 & 0.17 & 0.20 & 0.18 & 0.16 & 0.14 \\
\hline $\mathrm{MgO}$ & 7.53 & 7.09 & 1.49 & 5.55 & 10.32 & 5.52 & 5.84 & 8.47 \\
\hline $\mathrm{CaO}$ & 7.50 & 9.65 & 4.44 & 8.00 & 11.98 & 10.54 & 8.32 & 9.39 \\
\hline $\mathrm{Na} 2 \mathrm{O}$ & 4.50 & 3.98 & 5.06 & 3.02 & 2.01 & 3.71 & 4.16 & 3.51 \\
\hline $\mathrm{K} 2 \mathrm{O}$ & 3.50 & 1.11 & 6.80 & 5.55 & 3.42 & 4.71 & 3.84 & 3.00 \\
\hline P2O5 & 0.47 & 0.37 & 0.65 & 0.56 & 0.66 & 0.42 & 0.63 & 0.38 \\
\hline LOI & 3.74 & 6.61 & 1.89 & 1.64 & 2.13 & 0.82 & 3.22 & 2.85 \\
\hline Sum & 99.73 & 99.89 & 99.75 & 99.78 & 100.34 & 99.80 & 100.50 & 99.81 \\
\hline \multicolumn{9}{|l|}{ ppm } \\
\hline $\mathrm{Cr}$ & 455 & 207 & 14 & 204 & 280 & 61 & 191 & 505 \\
\hline $\mathrm{Ni}$ & 88 & 76 & 11 & 49 & 89 & 33 & 61 & 107 \\
\hline $\mathrm{Rb}$ & 56 & 83 & 171 & 150 & 92 & 96 & 45 & 36 \\
\hline $\mathrm{Sr}$ & 1652 & 432 & 2027 & 1523 & 1060 & 1478 & 2306 & 1302 \\
\hline $\mathrm{Zr}$ & 340 & 164 & 135 & 256 & 215 & 176 & 296 & 206 \\
\hline$Y$ & 21 & 21 & 20 & 25 & 20 & 22 & 24 & 19 \\
\hline $\mathrm{Nb}$ & 55 & 34 & 30 & 43 & 37 & 35 & 63 & 38 \\
\hline $\mathrm{Ba}$ & 2410 & 948 & 2520 & 1764 & 1050 & 1277 & 1313 & 1652 \\
\hline Th & 19 & 53 & 236 & 145 & 11.4 & 10 & 10 & 120 \\
\hline $\mathrm{La}$ & 154 & 72 & 88 & 106 & 89 & 75 & 105 & 60 \\
\hline $\mathrm{Ce}$ & 265 & 143 & 141 & 187 & 175 & 135 & 197 & 96 \\
\hline $\mathrm{Nd}$ & 104 & 73 & 53 & 74 & 76 & 62 & 69 & 30 \\
\hline $\mathrm{Sm}$ & 21 & 12.6 & 9.7 & 12.5 & 13.5 & 12.4 & 14 & 5.2 \\
\hline $\mathrm{Yb}$ & 3.3 & 1.6 & 1.9 & 1.9 & 2.1 & 1.9 & 2.2 & 0.7 \\
\hline $\begin{array}{l}\text { Lat } \\
\text { Long }\end{array}$ & $\begin{array}{l}25^{\circ} .48 .5^{\prime} \\
56^{\circ} 16.2^{\prime}\end{array}$ & $\begin{array}{l}25^{\circ} 52.8^{\prime} \\
56^{\circ} 16.3^{\prime}\end{array}$ & $\begin{array}{l}25^{\circ} 54.0^{\prime} \\
56^{\circ} 17.2^{\prime}\end{array}$ & $\begin{array}{l}25^{\circ} 53.0^{\prime} \\
56^{\circ} 15.0^{\prime}\end{array}$ & $\begin{array}{l}25^{\circ} 52.8 \\
56^{\circ} 14.0^{\prime}\end{array}$ & $\begin{array}{l}25^{\circ} 56.5^{\prime} \\
56^{\circ} 14.6^{\prime}\end{array}$ & $\begin{array}{l}25^{\circ} 57.0^{\prime} \\
56^{\circ} 05.3^{\prime}\end{array}$ & $\begin{array}{l}25^{\circ} 58.5^{\prime} \\
56^{\circ} 15.2^{\prime}\end{array}$ \\
\hline $\begin{array}{l}\text { Thickness } \\
\text { Direction }\end{array}$ & $\begin{array}{l}2.0 \mathrm{~m} \\
\mathrm{~N} 40 \mathrm{~W}\end{array}$ & $\begin{array}{l}0.25 \mathrm{~m} \\
\mathrm{~N} 80 \mathrm{~W}\end{array}$ & $\begin{array}{l}0.50 \mathrm{~m} \\
\text { N30E }\end{array}$ & $\begin{array}{l}3.50 \mathrm{~m} \\
\mathrm{~N} 50 \mathrm{~W}\end{array}$ & $\begin{array}{l}1.50 \mathrm{~m} \\
\mathrm{~N} 45 \mathrm{~W}\end{array}$ & $\begin{array}{l}2.20 \mathrm{~m} \\
\mathrm{~N} 42 \mathrm{E}\end{array}$ & $\begin{array}{l}2.0 \mathrm{~m} \\
\mathrm{~N} 45 \mathrm{~W}\end{array}$ & $\begin{array}{l}3.5 \mathrm{~m} \\
\mathrm{~N} 60 \mathrm{E}\end{array}$ \\
\hline mg\# & 0.658 & 0.732 & 0.476 & 0.571 & 0.662 & 0.557 & 0.578 & 0.667 \\
\hline A.I. & 1.022 & 0.613 & 0.910 & 0.805 & 0.508 & 0.762 & 0.759 & 0.776 \\
\hline $\mathrm{R}_{1}$ & 693 & 1103 & 279 & 756 & 1073 & 505 & 537 & 1061 \\
\hline $\mathrm{R}_{2}$ & 1391 & 1632 & 887 & 1399 & 2019 & 1690 & 1464 & 1652 \\
\hline
\end{tabular}


and three phonotephrites (sps. 1, 3 and 5). The average ${ }^{40} \mathrm{Ar} /{ }^{\beta 9} \mathrm{Ar}$ age is $126.6 \pm 1.1 \mathrm{Ma}$ (Comin-Chiaramonti et al., 2013).

The basanite dyke (potassic variant) shows porphyritic texture and contains seriate clinopyroxene $\left(\mathrm{Wo}_{45-48} \mathrm{En}_{42-47} \mathrm{Fs}_{8-12}\right)$, biotite (mg\# 0.70-0.74), and olivine $\left(\mathrm{Fo}_{74-84}\right)$ set in a

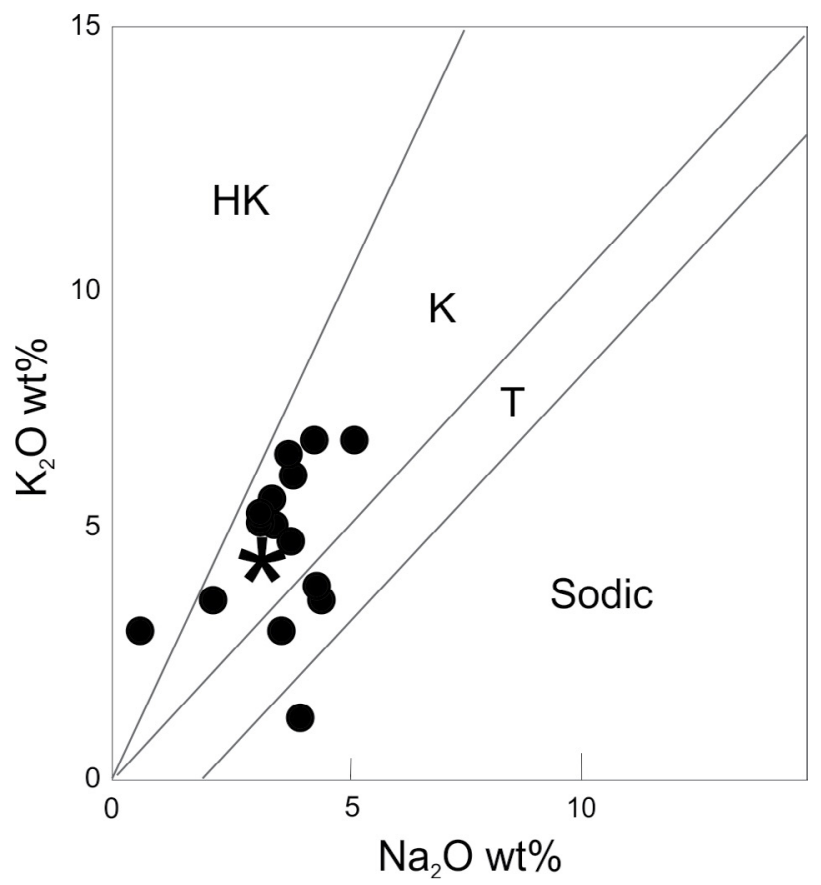

Figure 4. Discrimination diagram of $\mathrm{Na}_{2} \mathrm{O}$ vs. $\mathrm{K}_{2} \mathrm{O}$ (cf. Comin-Chiaramonti et al., 1996a) for the analyzed dykes. Symbols as in Figure 2. Distinguished fields are as follows: $\mathrm{HK}$, highly potassic; $\mathrm{K}$, potassic; $\mathrm{T}$, transitional; and $\mathrm{Na}$, sodic.

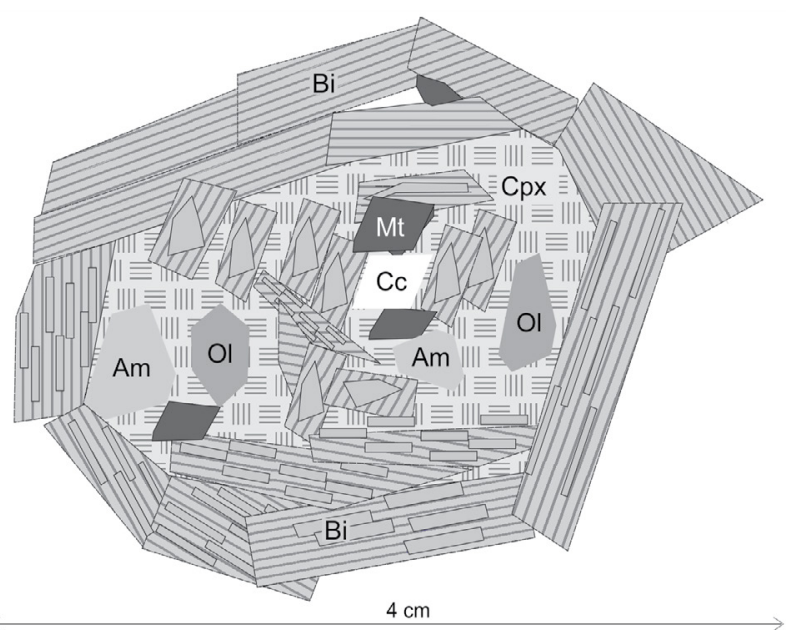

Figure 5. Textural relationships in the ocellus (sp. $2 \mathrm{~A}$ of Table 1) present in the trachyandesitic dyke 2. Legends: Am: amphibole; Bi: biotite; Cc: calcite; Cpx: clinopyroxene; Mt: magnetite; OI: olivine. hypocrystalline groundmass composed of clinopyroxene, mica, olivine, Fe-Ti oxides (ulvöspinel approximately $48 \mathrm{~mol} \%$ ) together with alkali feldspar and foids (similarly to lamprophyric minette, in conformity with Rock, 1987 and Le Maitre, 1989). Glass and carbonate patches are also recognized.

The tephrite dykes (potassic variant, sp. 12, and transitional variants, sps. 7 and 13) are strongly porphyritic. The ubiquitous phenocrysts and/or microphenocrysts consist of clinopyroxene $\left(\mathrm{Wo}_{42-47} \mathrm{En}_{40-44} \mathrm{Fs}_{9-14}\right)$, olivine $\left(\mathrm{Fo}_{72-79}\right)$ and pseudoleucite. Plagioclase $\left(\mathrm{An}_{20-70}\right)$, hastingsitic to pargasitic hornblende and Ti-magnetite (ulvöspinel up to $52 \mathrm{~mol} \%$ ) are found occasionally as phenocrysts and microphenocrysts. The groundmass is hypohyaline to hypocrystalline, possessing microlites of clinopyroxene $\left(\mathrm{Wo}_{47-48} \mathrm{En}_{38-40} \mathrm{Fs}_{12-15}\right)$, plagioclase $\left(\mathrm{An}_{14-20}\right)$, alkali feldspar $\left(\mathrm{Or}_{61-70}\right)$, Ti-magnetite, ilmenite, olivine, pseudoleucite, biotite, pargasite and apatite.

Phonotephrites (all potassic variants) are strongly porphyritic with microcrystalline to hypohyaline groundmass. Phenocrysts, microphenocrysts and microlites are composed of clinopyroxene $\left(\mathrm{Wo}_{46-48} \mathrm{En}_{38-40} \mathrm{Fs}_{12-15}\right)$, olivine $\left(\mathrm{Fo}_{71-79}\right)$, plagioclase $\left(\mathrm{An}_{14-65}\right)$, pseudoleucite, Ti-rich biotite, alkali feldspar, nepheline, Ti-magnetite (ulvöspinel varying from 38 to $50 \mathrm{~mol} \%$ ), apatite and zircon.

\section{AB-T suite (alkaline basalt to trachyphonolite/trachyte)}

As illustrated in Table 1 and Figure 2, the dykes of this suite are represented by two alkali basalts (sps. 8 and 14), three trachybasalts (sps. 6 and 10, plus the Mbocayaty sample), two trachyandesites (sps. 2 and 4) and one nepheline syenite (sp. 9; trachyphonolite as effusive equivalent). The average ${ }^{40} \mathrm{Ar} /{ }^{39} \mathrm{Ar}$ age is $125.2 \pm 3.8 \mathrm{Ma}$ (Comin-Chiaramonti et al., 2013).

The alkali basalts (transitional variant) are strongly porphyritic with plagioclase $\left(\mathrm{An}_{31-69}\right)$, clinopyroxene $\left(\mathrm{Wo}_{45-47} \mathrm{En}_{40-47} \mathrm{Fs}_{7-15}\right)$ and olivine $\left(\mathrm{Fo}_{69-83}\right)$ phenocrysts/ microphenocrysts, and Ti-magnetite microphenocrysts (ulvöspinel content up to $59 \mathrm{~mol} \%$ ). The hypocrystalline groundmass shows microlites of clinopyroxene $\left(\mathrm{Wo}_{45-46} \mathrm{En}_{38-39} \mathrm{Fs}_{15-17}\right)$, plagioclase $\left(\mathrm{An}_{25-30}\right)$, biotite (mg\# 0.65-0.70), alkali feldspar $\left(\mathrm{Or}_{70}\right)$, nepheline, Ti-magnetite, ilmenite, apatite and zircon.

The trachybasalts (potassic variants) exhibit porphyritic to strongly porphyritic texture. Phenocrysts and microphenocrysts include clinopyroxene $\left(\mathrm{Wo}_{4-46} \mathrm{En}_{41-48} \mathrm{Fs}_{8-14}\right)$, olivine $\left(\mathrm{Fo}_{56-77}\right)$, Ti-rich biotite (mg\# 0.56-0.65) and plagioclase $\left(\mathrm{An}_{40-61}\right)$ set in a hypohyaline to hypocrystalline groundmass, bearing microlites of clinopyroxene $\left(\mathrm{Wo}_{42-47} \mathrm{En}_{36-38} \mathrm{Fs}_{16-19}\right)$, plagioclase $\left(\mathrm{An}_{21-42}\right)$, alkali feldspar $\left(\mathrm{Or}_{50-70}\right)$, olivine $\left(\mathrm{Fo}_{48-56}\right)$, Ti-magnetite (ulvöspinel ranging from 41 to $55 \mathrm{~mol} \%$ ), apatite and zircon.

The trachyandesites (potassic variants) display a strongly porphyritic texture. Phenocrysts and microphenocrysts consist of clinopyroxenes $\left(\mathrm{Wo}_{48-49} \mathrm{En}_{35-40} \mathrm{Fs}_{12-17}\right)$, plagioclase 
$\left(\mathrm{An}_{40-50}\right)$, olivine $\left(\mathrm{Fo}_{46-57}\right)$, biotite, amphibole, opaques and apatite; additionally, large pseudoleucites (up to $10 \mathrm{~mm}$ across) were observed. The groundmass is hypohyaline with rare microlites of clinopyroxene, alkali feldspar, nepheline, opaques, apatite and zircon.

An ocellus is found in the trachyandesite (sp. 2, Figure 5) and is compositionally similar to that described by Comin-Chiaramonti et al. (2007a) in the ijolite sample from Cerro E Santa Elena. The ocellus is subcircular approximately $4 \mathrm{~cm}$ in diameter - and highly potassic and picrobasaltic in composition (Table 1 and Figure 2). Its outer rim is formed out of biotite $\left(\mathrm{mg} \# 0.70\right.$ and $\mathrm{K}_{2} \mathrm{O}$ $8.34 \mathrm{wt} \%$ ) and its center consists mainly of clinopyroxene (mg\# 0.82-0.99; $\left.\mathrm{Wo}_{48.5-48.0}, \mathrm{En}_{40.3-43.9}, \mathrm{Fs}_{11.7-8.1}\right)$. Subordinate

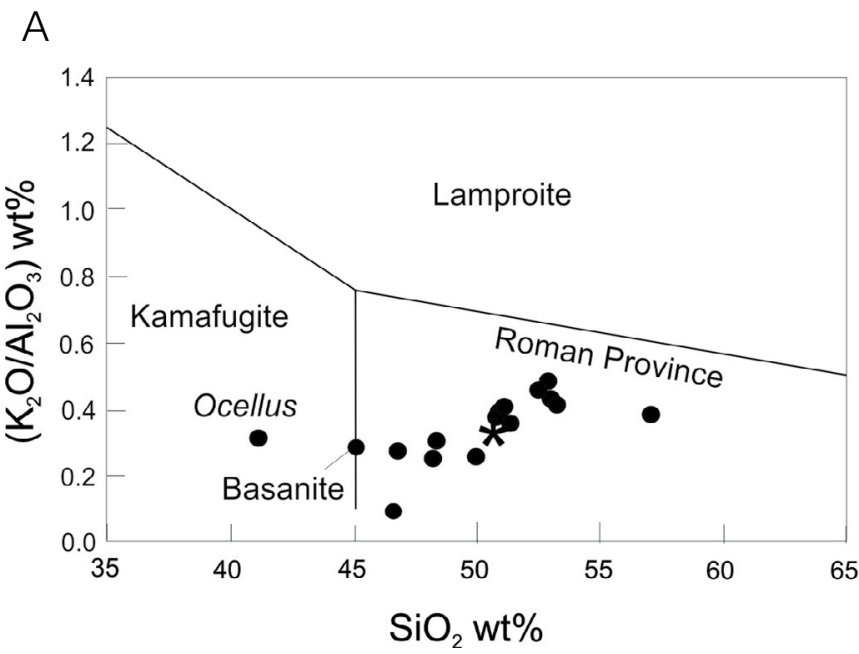

phases of the ocellus are olivine $\left(\mathrm{Fo}_{91}\right)$, amphibole $(\mathrm{mg} \# 0.8)$, magnetite (ulvöspinel $26 \mathrm{~mol} \%$ ) and calcite.

The nepheline syenite (potassic variant) is characterized by a holocrystalline seriate texture and contains alkali feldspar $\left(\mathrm{Or}_{60-77}\right)$, clinopyroxene $\left(\mathrm{Wo}_{47} \mathrm{En}_{30} \mathrm{Fs}_{23}\right)$, biotite, opaques (magnetite and ilmenite), nepheline $\left(\mathrm{Ne}_{84} \mathrm{Ks}_{8} \mathrm{wt} \%\right)$, and hastingsitic amphibole. It includes as accessory phases titanite, zircon and apatite (Comin-Chiaramonti and Gomes, 1996).

It is important to note that all the samples of Table 1 fall into the Roman Province field of Barton (1979) and Foley (1992) (Figures 6A and 6B), which is notably distinct from the Lamproite field. For this turn, the ocellus lies in the Kamafugite field, whereas the basanite (minette, sp. 11) lies at the boundary between the Roman Province and Kamafugite fields.

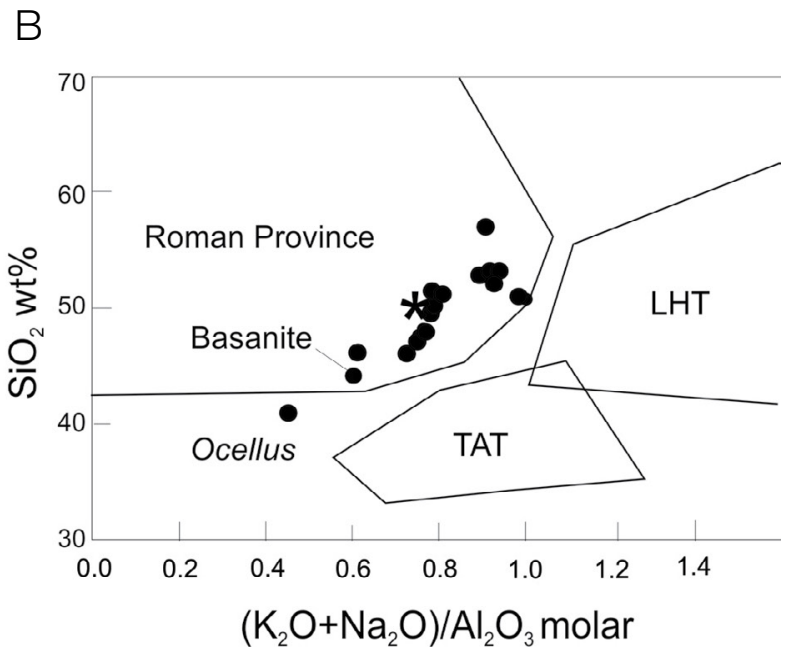

Figure 6. (A) Plot of $\mathrm{SiO}_{2}$ vs. $\mathrm{K}_{2} \mathrm{O} / \mathrm{Al}_{2} \mathrm{O}_{3}$. (B) $\mathrm{SiO}_{2}$ vs. molar $\left(\mathrm{K}_{2} \mathrm{O}+\mathrm{Na}_{2} \mathrm{O}\right) / \mathrm{Al}_{2} \mathrm{O}_{3}$ for the analyzed dykes. LHT and TAT, Leucite Hills and Toro Ankole provinces, respectively, according to Barton (1979), Foley (1992) and Bowen et al. (1998) and references therein.
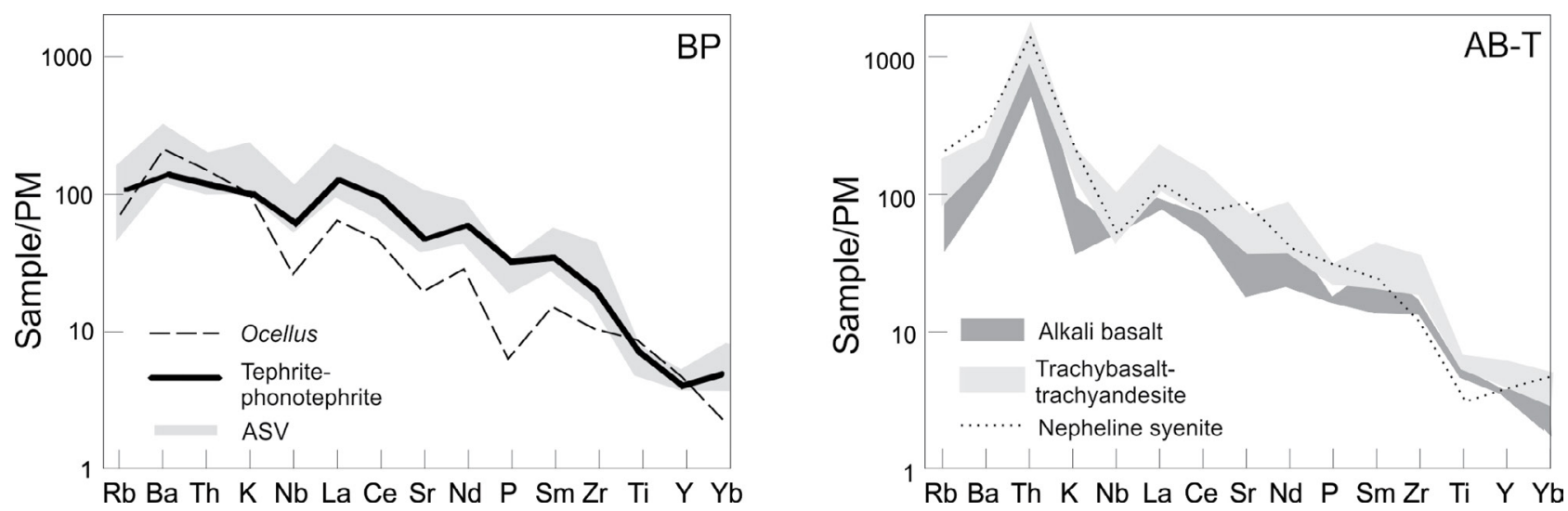

Figure 7. Primordial mantle (PM) trace element patterns for the Ybytyruzú dykes normalized according to Wood et al. (1979). The field for the ASV rocks is also shown. 


\section{Incompatible elements}

Normalized plots including certain REE data are show in Figure 7. In general, B-P and AB-T compositions have similar enrichment patterns for large-ion lithophile elements (LILE), i.e., $\mathrm{Rb}, \mathrm{Ba}, \mathrm{La}, \mathrm{Ce}$, and, secondarily, $\mathrm{Sm}$ and $\mathrm{Yb}$ (Comin-Chiaramonti et al., 1996a). In particular, the AB-T suite is characterized by a generally strong Th enrichment.

Finally, with regard to the behavior of the trace elements $\mathrm{Th}, \mathrm{Nb}$ and $\mathrm{Zr}$, the diagram $\mathrm{Nb}^{*} 100 / \mathrm{Zr}$ vs. Th*100/ $\mathrm{Zr}$ (Yilmaz, 2010) clearly indicates that the analyzed dykes are plotted within the Roman Province field (Figure 8) and showing no affinities with lamproitic rocks.

\section{CONCLUDING REMARKS}

The post-tholeiitic dykes from the Ybytyruzú region exhibit the same chemical variation as rocks associated with the Asunción-Sapucai-Villarrica graben. They can also be grouped into two main potassic suites, B-P (basanite-tephrite-phonotephrite-phonolite) and AB-T (alkali basalt-trachybasalt-trachyandesite-trachyphonolite/ trachyte). All the rock types show characteristics matching those of the Roman Province. Lamproitic dykes have not been recognized in the whole area and only a basanite outcrop may be classified to some extent as a lamprophyre (minette).

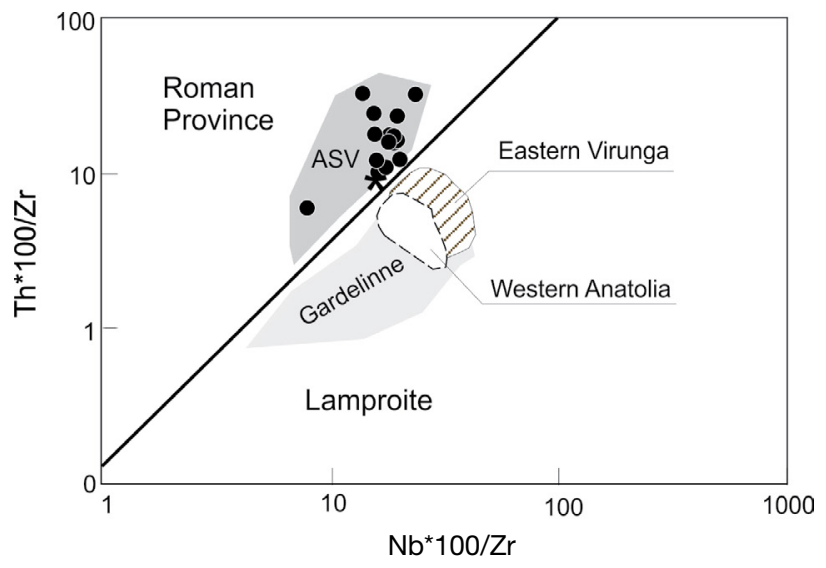

Figure 8. $\mathrm{Nb}^{\star} 100 / \mathrm{Zr}$ vs. $\mathrm{Th}{ }^{\star} 100 / \mathrm{Zr}$ (after Yilmaz, 2010) diagram displaying the fields of the Roman Province and some worldwide lamproites (Eastern Virunga, Rogers et al., 1998; Gardelinne, Chalapathi et al., 2016; Western Anatolia, Yilmaz, 2010). Symbols as in Figure 2. ASV represents the entire field of the potassic alkaline rocks from the Asunción-Sapucai- Villarrica graben.

\section{ACKNOWLEDGMENTS}

The authors are grateful to engineer E. Debernardi and to geologists D. Orué, L.A. Martinez and L. Lúcia for their collaboration in the field work. Thanks are due to the Brazilian agency Fapesp (Proc. 2013/18073-4, CBG) for financial support.

\section{REFERENCES}

Barton, M. (1979). A comparative study of some minerals occurring in the K-alkaline rocks of the Leucite-hills, Wyoming, the Vico Vulcano, Italy, and Toro-Ankola Region, Uganda. Neues Jahrbuch für Mineralogie Abhandlungen, 137, 113-134.

Bellieni, G., Brotzu, P., Comin-Chiaramonti, P., Ernesto, M., Melfi, A. J., Pacca, I. G., Piccirillo, E. M., Stolfa, D. (1983). Petrological and paleomagnetic data on the plateau basalt to rhyolite sequences of the Southern Paraná Basin (Brazil). Anais da Academia Brasileira de Ciências, 55, 355-383.

Bellieni, G., Comin-Chiaramonti, P., Marques, L. M., Martinez, L. A., Melfi, A. J., Nardy, A. J. U. R., Piccirillo, E. M., Stolfa, D. (1986). Continental flood basalts from the central-western regions of the Paraná plateau (Paraguay and Argentina): petrology and petrogenetic aspects: Neues Jarbuch für Mineralogie Abhandlugen, 154, 111-139.

Bergman, S. C. (1987). Lamproites and other potassium-rich igneous rocks: a review of their occurrences, mineralogy and geochemistry. In: J. G. Fitton, B. G. J. Upton (Eds.), Alkaline igneous rocks $(30,103-190)$. London: Geological Society.

Bowen, A., Pasteels, P., Punzalan, L. E., Yamba, T. K., Musisi, J. H. (1998). Quaternary perpotassic magmas in Uganda (Toro-Ankole Volcanic Province): age assessment and significance for magmatic evolution along the East African Rift. Journal of African Earth Sciences, 26, 463-476.

Chalapathi Rao, N. V., Atiullah, R., Alok, K., Samarendea, S., Purnendu, N., Ngazimpi, C., Lehmann, B., Rao, K. V. S. (2016). Petrogenesis of Mesozoic lamproite dykes from the Garledinne (Banganapalle) cluster, south-western Cuddapah Basin, southern India. Mineralogy and Petrology, 110, 247-268.

Comin-Chiaramonti, P., Censi, P., Cundari, A., De Min, A., Gomes, C. B., Marzoli, A., Piccirillo, E. M. (1996a). Petrochemistry of Early Cretaceous potassic rocks from the Asunción-Sapucai graben, central-eastern Paraguay. In: P. Comin-Chiaramonti, C. B. Gomes (Eds.). Alkaline magmatism in the central-eastern Paraguay. Relationships with coeval magmatism in Brazil (123-149). São Paulo: Edusp-Fapesp. 
Comin-Chiaramonti, P., Cundari, A., Bellieni, G. (1996b). Mineral chemistry of alkaline rock-types from the Asunción-Sapucai graben. Appendix III. In: P. Comin-Chiaramonti, C. B. Gomes (Eds.), Alkaline magmatism in the central-eastern Paraguay. Relationships with coeval magmatism in Brazil (389-458). São Paulo: Edusp-Fapesp.

Comin-Chiaramonti, P., Cundari, A., DeGraff, J. M., Gomes, C. B., Piccirillo, E. M. (1999). Early Cretaceous-Tertiary magmatism in Eastern Paraguay (western Paraná basin): geological, geophysical and geochemical relationships. Journal of Geodynamics, 28, 375-391.

Comin-Chiaramonti, P., Cundari, A., De Min, A., Gomes, C. B., Velázquez, V. F. (1996c). Magmatism in Eastern Paraguay: occurrence and petrography. In: P. Comin-Chiaramonti, C. B. Gomes (Eds.). Alkaline magmatism in the central-eastern Paraguay. Relationships with coeval magmatism in Brazil (p. 103-122). São Paulo: Edusp-Fapesp.

Comin-Chiaramonti, P., Cundari, A., Gomes, C. B., Piccirillo, E. M., Censi, P., De Min, A., Bellieni, G., Velázquez, V. F., Orué, D. (1992). Potassic dyke swarm in the Sapucai graben, Eastern Paraguay: petrographical, mineralogical and geochemical outlines. Lithos, 28, 283-310.

Comin-Chiaramonti, P., Cundari, A., Piccirillo, E. M., Gomes, C. B., Castorina, F., Censi, P., De Min, A., Marzoli, A., Speziale, S., Velázquez, V. F. (1997). Potassic and sodic igneous rocks from Eastern Paraguay: their origin from the lithospheric mantle and genetic relationships with the associated Paraná flood tholeiites. Journal of Petrology, 38, 495-528.

Comin-Chiaramonti, P., De Min, A., Cundari, A., Girardi, V. A. V., Ernesto, M., Gomes, C. B., Riccomini, C. (2013). Magmatism in the Asunción-Sapucai-Villarrica graben (Eastern Paraguay) revisited. Petrological, geophysical, geochemical and geodynamic inferences. Journal of Geological Research, 2013.

Comin-Chiaramonti, P., De Min, A., Gomes, C. B. (1996d). Magmatic rock-types from the Asunción-Sapucai graben: description of the occurrences and petrographical notes. Appendix I. In: P. Comin-Chiaramonti, C. B. Gomes (Eds.), Alkaline magmatism in the central-eastern Paraguay. Relationships with coeval magmatism in Brazil (275-330). São Paulo: Edusp-Fapesp.

Comin-Chiaramonti, P., De Min, A., Marzoli, A. (1996e). Magmatic rock-types from the Asunción-Sapucai graben: chemical analyses. Appendix II. In: P. Comin-Chiaramonti, C. B. Gomes (Eds.), Alkaline magmatism in the central-eastern Paraguay. Relationships with coeval magmatism in Brazil (331-388). São Paulo: Edusp-Fapesp.
Comin-Chiaramonti, P., Gomes, C. B. (Eds.). (1996). Alkaline magmatism in the central-eastern Paraguay. Relationships with coeval magmatism in Brazil. São Paulo: Edusp-Fapesp. 464p.

Comin-Chiaramonti, P., Gomes, C. B. (Eds.). (2005). Mesozoic to Cenozoic alkaline magmatism in the Brazilian Platform. São Paulo: Edusp-Fapesp. 752p.

Comin-Chiaramonti, P., Gomes, C. B., De Min, A., Ernesto, M., Marzoli, A., Riccomini, C. (2007a). Eastern Paraguay: an overview of the post-Paleozoic magmatism and geodynamic implications. Rendiconti Lincei, Scienze Fisiche e Naturali, 18, 139-192.

Comin-Chiaramonti, P., Marzoli, A., Gomes, C. B., Milan, A., Riccomini, C., Mantovani, M. M. S., Renne, P., Tassinari, C. C. G., Vasconcelos, P. M. (2007b). Origin of Post Paleozoic magmatism in Eastern Paraguay.The Geological Society of America, Special Paper, 430, 603-633.

De La Roche, H., Leterrier, J., Grandclaude, P., Marchal, M. (1980). A classification of volcanic and plutonic rocks using R1-R2 diagram and major-element analyses: its relationships with current nomenclature. Chemical Geology, 29, 183-210.

Ernesto, M., Comin-Chiaramonti, P., Gomes, C. B., Castillo, A. M. C., Velázquez, J. C. (1996). Palaeomagnetic data from central alkaline province, Eastern Paraguay. In: P. Comin-Chiaramonti, C. B. Gomes (Eds.), Alkaline magmatism in the central-eastern Paraguay. Relationships with coeval magmatism in Brazil (85-102). São Paulo: Edusp-Fapesp.

Foley, S. F. (1992). Petrological characterization of the source components of potassic magmas: geochemical experimental constraints. Lithos, 28, 187-204.

Gomes, C. B., Comin-Chiaramonti, P., De Min, A., Melfi, A. J., Bellieni, G., Ernesto, M., Castillo, A. M. C., Velázquez, V. F. (1989). Atividade filoniana associada ao complexo alcalino de Sapukai, Paraguai Oriental. Geochimica Brasiliensis, 3, 93-114.

Gomes, C. B., Comin-Chiaramonti, P., Velázquez, V. F. (2000). The Mesoproterozoic rhyolite occurrences of Fuerte Olimpo and Fuerte San Carlos, northern Paraguay. Revista Brasileira de Geociências, 30, 785-788.

Gomes, C. B., Comin-Chiaramonti, P., Velázquez, V. F. (2013). A synthesis on the alkaline magmatism of Eastern Paraguay. Brazilian Journal of Geology, 43, 745-761.

Le Maitre, R. W. (1976). Some problems of the projection of chemical data into mineralogical classification. Contributions to Mineralogy and Petrology, 56, 181-189. 
Le Maitre, R. W. (Ed.). (1989). Igneous rocks: a classification and glossary of terms. Oxford: Blackwell Scientific Publications. 193p.

MacDonald, R. (1974). Nomenclature and petrochemistry of the peralkaline oversaturated extrusive rocks. Bulletin of Volcanology, 38, 498-516.

Mitchell, R. H., Bergman, S. C. (Eds.). (1991). Petrology of lamproites. New York: Plenum Press. 447p.

Piccirillo, E. M., Melfi, A. J. (Eds.). (1988). The Mesozoic flood volcanism from the Paraná Basin (Brazil). Petrogenetic and geophysical aspects. São Paulo: IAG-USP. 600p.

Presser, J. L. B., Bitschene, P. R., Vladykin, N. V. (2014). Comentarios sobre la geologia, la petrografia y la quimica mineral de algunas lamproitas de la porciòn norte de la Cordillera del Ybytyruzú, Paraguay Oriental. Boletim del Museo Naional de Historia Natural del Paraguay, 18, 24-61.

Renne, P. R., Ernesto, M., Pacca, I. G., Coe, R. S., Glen, J. M., Prevot, M., Perrin, M. (1992). The age of Paraná flood volcanism, rift of Gondwanaland, and Jurassic-Cretaceous boundary. Science, 258, 975-979.

Renne, P. R., Mertz, D. F., Teixeira, W., Ens, H., Richards, M. (1993). Geochronological constraints on magmatic and tectonic evolution of the Paraná Province. EOS, American Geophisical Union, 74, 553.

Rock, N. M. S. (1987). The nature and origin of lamprophyres. Geological Society, 30, 191-226.
Rogers, N. W., James, D., Kelley, S. P., De Mulder, M. (1998). The generation of potassic lavas from the Eastern Virunga Province, Rwanda. Journal of Petrology, 39, 1223-1247.

Velázquez, V.F., Gomes, C. B., Capaldi, G., Comin-Chiaramonti, P., Ernesto, M., Kawashita, K., Petrini R., Piccirillo, E. M. (1992). Magmatismo alcalino mesozóico na porção centro-oriental do Paraguay: aspectos geocronológicos. Geochimica Brasiliensis, 6, 23-35.

Velázquez, V. F., Riccomini, C., Gomes, C. B., Kirk, J. (2011). The Cretaceous alkaline dyke swarm in the central segment of the Asuncion Rift, Eastern Paraguay: its regional distribution, mechanism of emplacement and tectonic significance. Journal of Geological Research, 2011. DOI: http://dx.doi.org/10.1155/2011/946701

Wood, D. A., Joron, J. L., Treuil, M., Norry, M. J., Tarney, J. (1979). Elemental and Sr-isotope variations in basic lavas from Iceland and the surrounding ocean floor. Contributions to Mineralogy and Petrology, 70, 319-339.

Woolley, A. R., Bergman, S., Edgar, A., Le Bas, M., Mitchell, R., Rock, N. M. S., Scott Smith, B. H. (1996). Classification of lamprophyres, lamproites, kimberlites and the kalsilitic, melilitic and leucititic rocks. Canadian Mineralogist, 34, 175-186.

Yilmaz, K. (2010). Origin of anorogenic "lamproite-like" potassic lavas from the Denizli region in Western Anatolia Extensional Province, Turkey. Mineralogy and Petrology, 99, 219-239. 